\title{
Lowering the Barriers to Cancer Imaging
}

\author{
M.S. Avila-Garcia ${ }^{1}$, A. E. Trefethen ${ }^{1}$, M. Brady ${ }^{2}$, F. Gleeson ${ }^{3}$ and D. Goodman ${ }^{1}$ \\ ${ }^{I}$ Oxford e-Research Centre, University of Oxford, UK \\ ${ }^{2}$ Department of Engineering Science, University of Oxford, UK \\ ${ }^{3}$ Radiology, Nuffield Dept. of Surgery, Churchill Hospital, University of Oxford, UK \\ susana.garcia@oerc.ox.ac.uk; anne.trefethen@oerc.ox.ac.uk; jmb@robots.ox.ac.uk; \\ fergus.gleeson@nds.ox.ac.uk; daniel.goodman@oerc.ox.ac.uk;
}

\begin{abstract}
There are various issues that limit the development and deployment of new software solutions in cancer image analysis research. In this paper we discuss some of these and propose a framework design based on cloud computing concepts, Microsoft technologies, existing middleware and imaging toolkits. Furthermore, we address some of these issues by introducing collaborative visual tools for visual input data and multi-user interactions.
\end{abstract}

\section{Introduction}

Approximately 36,000 people are diagnosed with colorectal cancer every year in UK which makes it the third most common cancer in UK [3]. Furthermore, colorectal cancer often metastasizes to the liver with poor prognosis, and liver cancer itself causes around 3,000 deaths each year in the UK [4]. Medical imaging techniques such as magnetic resonance imaging (MRI), ultrasound (US), computerized tomography (CT) and a combination of positron emission tomography (PET) with CT (PET/CT), have been used for detecting, staging, and monitoring the evolution of patients with colorectal and liver cancer. Radiologists analyze medical images to detect abnormalities, and when one of these is categorized as a tumor it has to be characterized; its size, location and configuration provide information to support the prognosis. Image segmentation and registration are keys to such a categorization and to support the decision-making for treatment delivery and the response analysis by comparing tumor shape, location and volume at different time periods. However, colorectal cancer images are often noisy, complex and highly textured and the segmentation of tumors is challenging due to the poor contrast relative to their surroundings.

The types of segmentation and registration algorithms that are suitable for any given image will depend on several parameters, and researchers are continually working to improve on existing algorithms. When a new project starts a researcher must consider all existing solutions to determine if they are suitable or not. This may be very time consuming and may not lead to relevant algorithms that are implemented in a way that the researcher can adopt the solution.

On the other hand, clinicians need to become familiar with the software solutions developed by MIA researchers in order to provide feedback about their performance as well as to generate data for validating the results obtained.

In this paper we will describe some of the difficulties that medical image analysis researchers and clinicians face and introduce our approach to improve their efficiency capability.

\section{Working practice at Oxford}

Medical image analysis (MIA) researchers at Oxford are working with clinicians at John Radcliffe and Churchill Hospitals on image analysis of colorectal and liver cancer images. We interviewed MIA researchers and clinicians in order to identify the issues limiting their research work. The issues MIA researchers deal with are, but not limited to:

a) Implementing methods developed by other researchers. This can be both very laborious and time consuming; they spent around $30 \%$ of their research time doing so. An instance that difficult this task is the omission of implementation details in the description of the algorithm and when an implementation is achieved there may be no way to validate it. 
b) Re-using code developed by other researchers. When an algorithm is available as a piece of software the researcher might find difficult to use it as it is defined in a programming language that he/she is not familiar with, that is no longer supported by modern compilers or that is simply not compatible with their own development environment.

c) Using imaging and visualization toolkits bound up in specific languages. Researchers must take the toolkit as a whole, or not use it. Adapting or adding new algorithms require specialized programmer skills.

d) Deploying solutions for clinical use. Additional time should be invested in order to develop suitable user interfaces for clinicians.

e) Generating ground truth. The generation of examples with target shapes, formally called ground truth, which are manually outlined by either clinicians or MIA researchers could be both tedious and time consuming.

On the other hand, clinicians face the following issues:

a) Analyzing and processing images in increasingly advanced ways. They reach a frustratingly low limit to what they can do with reasonable effort with no specialist programmer's support.

b) $3 D$ and advance visualization of tumors during multidisciplinary team meetings. Although there are sophisticated visualization tools often available in picture archiving computer systems (PACS), clinicians do not have such functionality to visualize and display tumors during multidisciplinary team (MDT) meetings, where cases of colorectal and liver cancer are discussed.

Besides, the interaction among these specialists, both clinicians and MIA researchers, is limited by the visual tools they use to analyze images and discuss their results. We investigated which are the development tools MIA researchers are familiar with. MAtLAB ${ }^{\circledR}$ is used by all researchers as a fast prototyping tool, while 6 of them use $\mathrm{C}++$ as well, as shown in table 1. This is often used to re-implement MATLAB ${ }^{\circledR}$ code in order to improve its computational performance. One researcher uses Java to develop his algorithms and ImageJ to display results.

The image toolbox from MatLaB ${ }^{\circledR}$ is the most popular imaging toolkit, as shown in table 2 , followed by the insight segmentation and registration toolkit (ITK). The majority of $\mathrm{C}++$ users work under
Windows platforms, using Microsoft Visual Studio and two of them work under a Linux platform using gec.

The needs mentioned above outline the requirements for the framework MIA researchers need as:

a) A platform independent framework.

b) A repository of algorithms they can share with no bounds to specific programming languages.

c) Unbound up already existing imaging and visualization toolkits in specific programming languages.

d) Access to the most up-to-date authoritative knowledge.

e) A framework for rapid development and deployment of applications for clinical use.

f) Improve mechanisms for manual segmentation

Table 1. Programming languages used by interviewed MIA researchers.

\begin{tabular}{|c|c|}
\hline Programming Language & Number of Users \\
\hline MATLAB ${ }^{\circledR}$ & 7 \\
\hline C++ & 6 \\
\hline Java & 1 \\
\hline
\end{tabular}

Table 2. Toolkits used by interviewed MIA researchers.

\begin{tabular}{|c|c|}
\hline Toolkit & Number of Users \\
\hline $\begin{array}{c}\text { MATLAB }{ }^{\circledR} \text { Imaging } \\
\text { Toolkit }\end{array}$ & 7 \\
\hline ITK & 3 \\
\hline VTK & 1 \\
\hline SPM & 1 \\
\hline Volview & 1 \\
\hline Image J & 1 \\
\hline
\end{tabular}

\section{Use case scenario}

The basic use case scenario defined is based on a collaborative environment where methods can be shared, plugged in to new applications with minimum effort, and no programming language bindings.

The ultimate end users of this collaborative environment are both MIA researchers and clinicians. The main tasks MIA researchers can perform are:

a) Searching for existing methods and solutions.

b) Accessing to publications and experimental data for a specific solution.

c) Reviewing experimental findings.

d) Computational steering of existing solutions. 
e) Evaluating solutions/methods using their own data.

f) Interconnecting various methods to generate inbox solutions. For instance, a basic image analysis application may consist in interconnecting blocks for reading an image in a specific format, pre-processing it in order to improve its quality, i.e. filtering, applying a segmentation algorithm, visualizing results, and documenting findings. Each of these actions can be categorized as methods/blocks that can be plugged and played in new applications.

g) Analyzing and visualizing results. Provide access to visualization tools to enable the analysis of results. Providing mechanisms to document such results, and to generate the associated metadata.

h) Sharing results with other colleagues and clinicians. MIA researchers are able to test the solution and provide suggestions, concerns, etc. Clinicians are able to analyze and visualize results and send the appropriate feedback to MIA researchers.

i) Looking for appropriate user interface tools. Have access to user interfaces developed in the past and re-use those tools needed. Associated metadata will provide information of which tools have being used in specific applications.

j) Sending requests to generate ground truth information to a clinician.

k) Deploying applications for clinical use. Applications with low demands on interaction and advanced visualization could be deployed as web services. This enables remote collaboration among researchers and clinicians.

1) Reviewing logs and data to support the publication and dissemination of results. Generating logs and the corresponding links to experiments and results provide a better mechanism for gathering information and facts to be published.

m) Changing access permissions to share resources. Initially only members of the project will have access to associated resources. Researchers then define whether such resources can be shared with other researchers or not.

On the other hand, clinicians will be able to perform the following tasks:

a) Generation of ground truth and its deployment. Metadata associated with the segmented shapes, and additional annotations are generated. Automatic notifications are then sent to the associated MIA researchers. b) Evaluation of applications. Proving feedback to the corresponding MIA researchers about the software solutions developed.

c) Participating in discussion forums.

This scenario would improve general interaction among clinicians and researchers as well as the dynamics while testing and evaluating software solutions. However, there are a number of existing solutions proving similar functionality in other scenarios.

\section{Related activities}

There are a number of e-Science projects in medical and health care research in the UK that address similar issues which have been built on top of other eScience projects based on grid technologies and workflows. The integrative biology project provides a collaborative "in silico" framework for computational biologist to perform experiments in a distributed environment enabled by Grid technologies [7]. This project supports the simulation of human organs at cellular and molecular level for the development of new drugs and has been built up on the top of different projects such as myGrid, gViz, Godiva, RealityGrid and Geodise. MyGrid provides support for "in silico" experiments through the use of workflows using the Taverna workbench [9], which has become a building block for many projects including the Carmen project; which provides a virtual research environment (VRE) for neuroscientists [20]. Neurogrid also provides an imaging toolkit for neuroscience applications [8].

Research Information Centre, RIC, is a VRE developed by the Technical Computing Group at Microsoft and The British Library to support researchers in managing the range of tasks involved in carrying out research [1]. The implementation of the RIC is focused on biomedical research. MyExperiment is a VRE inspired on Web 2.0 that enables researchers to share and execute workflows [14].

Various projects use Grid technologies in order to provide access to remote resources and to speed up image analysis. For instance, the IXI project delivered a framework to facilitate the analysis of MR images and performing image registration and segmentation tasks [19]. eDiamond [2] uses grid technology to support breast cancer research whilst GIMI (Generic Infrastructure for Medical Informatics), provides middleware to support data and service sharing for health care training [16].

The projects mentioned above overcome the issue of moving data across the network and executing expensive computational tasks by developing the 
middleware infrastructure and some of them provide an end-user interface which hides the underlying complexity of accessing remote computational services. Some of them make use of graphical programming tools such as workflows. These have become popular programming tools and various workbenches have been developed in the past few years. Some of the most popular are Taverna, Triana and Kepler.

Taverna is an open source scientific workflow workbench developed to support "in silico" experiment for bioinformaticians[17]. Workflows, which can be written in a new language called the simple conceptual unified flow language (SCUFL), are generated within a graphical tool environment with access to different types of processes e.g. WSDL and Soaplab types. Taverna is being used in different e-Science projects such as MyExperiment and Carmen.

On the other hand, Microsoft Workflow Foundation is the programming model and engine for building workflow enabled applications on Windows. It consists of .NET classes, which are part of the Microsoft .NET Framework 3.0, an in-process workflow engine, and designers for Visual Studio[13].

There are other approaches such as SciRun and IRIS Explorer, which provide the use of graphical interface and remote resources; they are not documented to support web services yet though. IRIS explorer is a tool for developing visualisation applications that was developed using different libraries including OpenGL, ImageVision and NAG's numerical libraries [21].

Although workflows are useful for researchers, they are complex to understand for non IT people such as clinicians. Therefore deploying applications for clinical use require an additional work. These problems were addressed by researchers in the Scientific Computing and Imaging (SCI) Institute of the University of Utah when they deployed an application developed in the SCIRun environment. It was difficult for clinicians to understand the way the methods worked and the standards used by researchers, such as the colour palette used to represent data in a graph or an image. SCIRun is an open source toolkit that offers an environment for scientific computation and visualisation using a dataflow interface [15]. Although this interface is straightforward to use for researchers it was not for clinicians, partly because the representation of the information had different interpretations among researchers and clinicians and because clinicians, need to define parameters which are not very intuitive. Therefore, scientific applications called PowerApps were incorporated in SCIRun, making applications more usable by clinicians. Bioimage, one of those
powerApps, provides image processing and 3D visualisation tools.

There are various imaging toolkits available to facilitate image analysis research. An approach that provides segmentation and registration methods is the Insight Segmentation and Registration toolkit (ITK), an open source software system that was developed to support organ segmentation of images generated by the Visible Human project [10]. This has become a toolkit widely used by image analysis researchers. An imaging researcher may contribute to this repository by deploying methods following certain procedures. There are some tools developed using this toolkit to perform semiautomatic segmentation such as ITK SNAP [22].

MATLAB ${ }^{\circledR}$ is well known for its fast prototyping characteristics and its image processing toolbox is often used by imaging researchers in order to create their algorithms and to display their results. Usually MATLAB ${ }^{\circledR}$ is used when the aim is to proof a concept and not to write an optimized program. As MATLAB ${ }^{\circ}$ is a widely used, an interface to use ITK algorithms under this environment has been reported in [5] as MatITK. However, in terms of image visualisation MATLAB $®$ does not offer extensive functionality and imaging researchers have to look for new tools to display and visualise their results. VTK is a toolkit popular among imaging researchers for $3 \mathrm{D}$ visualisations, which is an open source library, developed in $\mathrm{C}++$ with interface layers including, Tcl/Tk, Java, and Python [11].

The extensible imaging platform (XIP) is an open source environment that provides a set of visual 'drag and drop' programming tools for the rapid development of imaging and visualization applications which also provides a friendlier environment for utilizing popular toolkits such as ITK and VTK [18].

\section{Cloud computing framework for cancer imaging analysis research}

In order to fulfil the requirements for our use case, we propose the design of a framework to maximize the efficiency of a medical image analysis researcher by considering:

a) Efficient access to the most appropriate data sources.

b) A set of increasingly specialized processes and algorithms relevant to his/her needs.

c) Efficient access to the most up-to-date, authoritative knowledge that can serve as metadata.

d) A framework to support the orchestration, choreography and workflow of image capture, adaptation, annotation and distribution. 
e) An effective way to work with like-minded specialists, possibly working in different time zones.

f) Dynamic mobilization of computational imaging services that are currently "looked up" in imaging and visualization toolkits.

g) Collaborative visual tools (including multitouch and interactive surfaces) for multi-user and visual data input.

Our goal is to develop a framework that takes the best characteristics of the solutions presented above to offer an integral solution with the following considerations:

a) Provide a VRE as a collaboration framework among MIA researchers and clinicians.

b) Provide a problem solving environment (PSE) based on workflows, to enable rapid application development.

c) Provide access to imaging and visualization toolkits as web services.

Therefore, we will develop a use case to plug it into a virtual research environment (VRE), which can provide the use case functionality described above, and overriding considerations for:

a) Data provenance, where contributions of each researcher are registered and the use of their methods and experimental data is acknowledged.

b) Various levels of information access to provide security and data confidentiality when needed.

c) Manage the concept of experiments where links to various objects can lead the researcher to the information required.

d) Provide discussion forums to enable communication and collaboration among researchers.

Although, a cloud computing framework will allow the use of remote computing services with platform independent access and complexity hidden underneath, so that the access to web services is transparent for the ultimate end-users, a web-based approach would not allow them to fully interact with image analysis applications as it may be required. Therefore, we propose a solution by considering:

a) Portal to enable a platform independent environment to access remote research services via web services.

b) Desktop enriched application for applications demanding interactive interfaces and advanced visualization.

The use of workflows usually facilitates the orchestration of new application with a minimum effort. Most of MIA researchers are familiar with Microsoft technologies, including the Visual Studio programming environment. Therefore we will take advantage of that and will use them to develop our enriched application. We will use SQL Server as our database platform and Microsoft Workflow foundation as our workflow engine. Given previous investments in similar solutions it makes sense for us to adopt and adapt existing software environments.

However, all these considerations undercover great challenges to be faced.

\section{Challenges}

There are various challenges for this project, which include:

a) Adapting existing software solutions to our use case. Although requirements for our environment look very similar to those developed in other scenarios, an exhaustive analysis of our use case should conduct to the software that provides a best approach to the functionality we are looking for. However, adaptation of those environments may require significant time.

b) Encourage researchers to share their data and resources. Sharing should be appreciated as a dissemination process.

c) Unbound up toolkits from specific languages. One of the major issues is license management for commercial solutions as MATLAB $®$, in which case we will explore the use of the MatLaB ${ }^{\circledR}$ Builder ${ }^{\mathrm{TM}}$ NE for Microsoft ${ }^{\circledR}$.NET framework.

d) Provide friendly user interfaces and mechanisms for researchers to deposit their contributions. Although there must be mechanisms to control the quality of such deployments those should be considered to facilitate such a procedure.

e) Design and development of suitable user interfaces for clinicians. Create a toolbox with user interface controls and tools that researchers can use for deploying their applications. However, the development of user interfaces might take considerable time.

f) Enhancement of collaboration and interaction among MIA researchers and clinicians. When thin applications can be deployed to be executed in a portal, clinicians should be able to provide feedback within such environment.

g) Promote the access to engineering and computer science academics, and 
undergraduate students, to raise interest in challenges to solve computational and software engineering problems, e.g. re-define methods for high performance computing, improve computational costs of specific algorithms, etc. This would both allow and encourage students to be involved with real problems and propose real solutions.

h) Enable access to medical science academics and students to engage them with the use of image processing techniques in an earlier stage, as well as to collaborate with students and researchers of different backgrounds.

i) Link to the Oxford University Research Archive (ORA), a repository for research materials produced by scholars at Oxford University, to ensure access to a permanent and secure online archive.

\section{Work Packages}

We have defined a number of work packages for this project:

- Work Package 0 (WP0) is concerned about the analysis and adoption of existing software solutions and environments.

- Work Package 1 (WP1) involves the design of standard datasets, metadata specification and the definition of web services for medical analysis algorithms.

-Work Package 2 (WP2) will tackle the problem of developing applications and "reusing" existing code by means of Windows Workflow Foundation.

-Work Package 3 (WP3) will incorporate existing imaging and visualisation toolkits as web services.

-Work Package 4 (WP4) will explore the mechanisms for clinicians to use applications developed and to contribute to the project by providing feedback. The development of a set of tools for user interface development could be required.

-Work Package 5 (WP5) is concerned about ground truth data generation, by manual segmentation, and the enhancement of interaction and collaboration among MIA researchers and clinicians by means of multitouch and interactive visual tools.

- Work Package 6 (WP6) will be concern to enable links to permanent and online repositories of research publications such as ORA.

\section{Multi-touch and interactive surfaces to enhance Cancer imaging (WP5)}

The use of collaborative visual tools for multi-user and visual data input provides an approach to enhance interaction and collaboration among researchers and clinicians. The use of specialized jargon may be a barrier for mutual understanding among clinicians and MIA researchers, whilst for more homogeneous teams, which share the same background, such interactions might be easier. However, the visualization of an image and pointing to the characteristics of interest may improve the mutual understanding among specialists with different backgrounds.

While discussing results in a meeting, participants usually gather around a computer screen that displays the results as the use of other displays and printers might affect the image contrast and resolution of the images and usually user must take turns in order to manipulate the corresponding images.

For instance, in MDT meetings each case of colorectal and liver cancer is discussed in order to make decision about the treatment delivery. Cancer images are displayed by a projector on a wall. Each case is prepared prior to the meeting in order to present the relevant information to an audience of radiologists, pathologists, oncologists, surgeons and specialized nurses. Figure 1(a) shows an example of how the attendees are distributed within the meeting room The radiologist presenting the analysis of the images is the one in charge of manipulating the images and showing the findings to an audience of often more than 15 participants. We believe that these interactions might be enhanced by the use of multi-touch technologies with a combined set-up of vertical and horizontal displays. Horizontal for active members, who are those participating in the decision making, and a vertical display for passive members, those attending to learn from such discussions, as illustrated in Figure 1(b).

In terms of validating image segmentation solutions, it may be required by MIA researchers the generation of ground truth information. This task has been enhanced in terms of time and accuracy by the use of graphics tablet technology, which use is illustrated in Figure 2.

We believe that the use of multi-touch and interactive surfaces, such as the Mitsubishi DiamondTouch table [6] and the Microsoft Surface Computer [12], will provide a more interactive way to perform such a task as both MIA researchers and clinicians will be able to manipulate and visualise 
images in a large and interactive display and to outline shapes of interest using their own fingers.

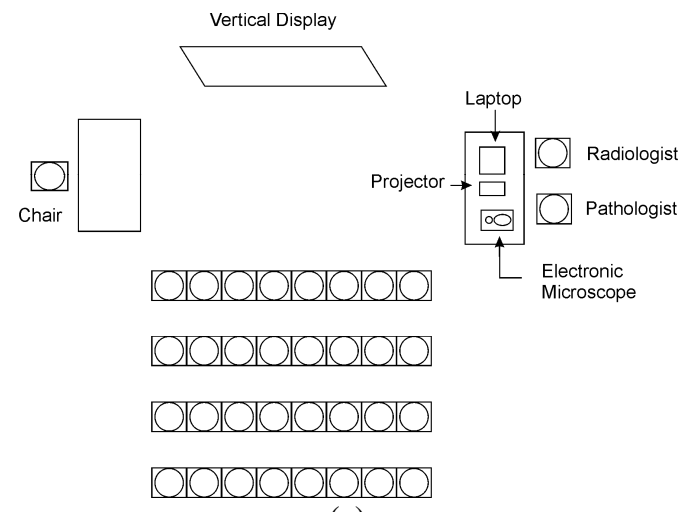

(a)

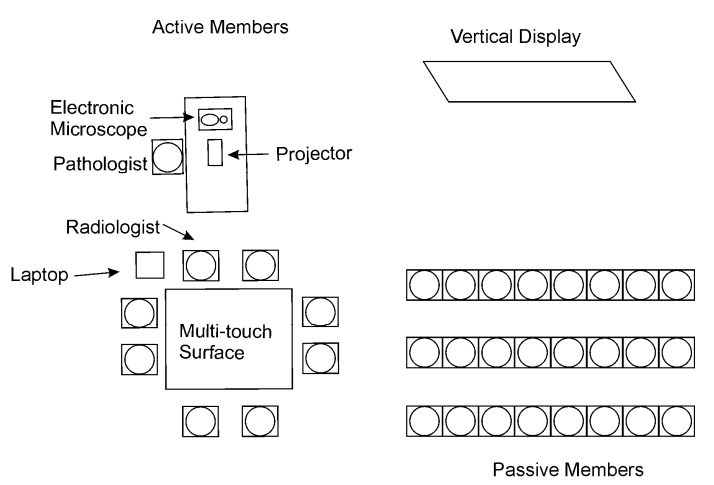

(b)

Figure 1. (a) Illustration of how MDT members are currently distributed, (b) proposed configuration for the use of multi-touch surfaces during MDT meetings

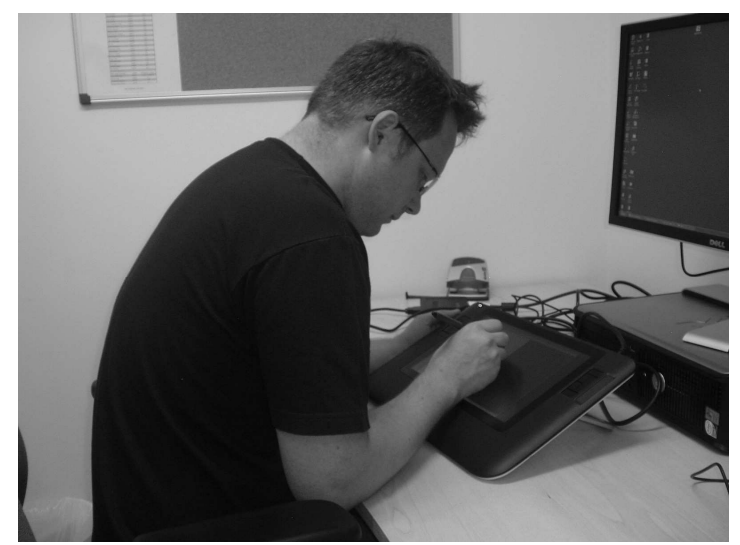

Figure 2. Illustration of the use of a Cintiq 12WX Wacom graphics tablet for manual segmentation.

\section{Summary and future work}

We have presented some of the issues MIA researchers and clinicians deal with. Although there is a large number of imaging and toolkits, they are bound up in specific languages that the user must take as a whole or not use, and adding and adapting requires specialized programmer skills.

We have proposed a framework design based on cloud computing concepts and toolkits that are already popular among MIA researchers to maximise the efficiency of researchers and clinicians on the development, deployment, and evaluation of medical image analysis applications for clinical use. We will use, where possible, Microsoft tools such as SQL server as database manager, Windows Workflow Foundation as our workflow engine and the Microsoft .Net framework to develop our web services and applications. We will also look for existing software environments that can contribute and fit within our use case with support to existing imaging and visualization toolkits. This framework will alleviate the frustration of MIA researchers spending significant research time implementing methods reported and developed by other researchers, instead it will promote collaboration among researchers allowing easier access to existing work.

Although researchers are enthusiastic about accessing code and research data of other researchers, some of them find difficult to share information and code. If sharing is addressed as a dissemination process then researchers would be more enthusiastic about such an idea.

Challenges for this project include the enhancement of MIA researchers and clinicians interactions. We will assess the use of multi-touch technology as a subtly and intuitive way to engage clinicians with the use of new medical image analysis applications as well as for enhancing the way medical image analysis researchers and clinicians interact.

\section{Acknowledgments}

This research is funded by the Technical Computing Initiative of Microsoft Corporation. We thank participants involved within the evaluation of graphics tablets for the generation of ground truth and especially Adi Himpson. We also thank MIA researchers at Oxford for their valuable comments during the analysis of requirements for this project, especially Vicente Grau and Niranjan Joshi, as well as radiologists working at Churchill and John Radcliffe Hospitals.

\section{References}


[1] Barga R.S., Andrews S. and Parastatidis S. A Virtual Research Environment (VRE) for Bioscience Researchers. ADVCOMP, 2007.pp 31-38. 2007.

[2] Brady M., Gavaghan D., Simpson A., Highnam R., Mulet M. "eDiamond: Digital Mammography". Proceedings of UK e-Science All Hands Meeting 2003. Nottingham, UK. 2003.

[3] CANCER RESEARCH UK (2008). "Cancer Research UK: CancerStats key facts on bowel cancer" [online]. [cited August 5, 2008].

<http://info.cancerresearchuk.org/cancerstats/types/bowel/>.

[4] CANCER RESEARCH UK (2008). "Cancer Research UK: UK Liver cancer statistics" [online]. [cited August 5, 2008].

$<$ http://info.cancerresearchuk.org/cancerstats/types/liver/?a=5 $441>$

[5] Chu V. and G. Hamarneh. "MatLAB-ITK Interface for Medical Image Filtering, Segmentation, and Registration" Proceedings of SPIE Medical Imaging: Image Processing, vol. 6144, 2006, pp. 1-8 (61443T-1 to 8).

[6] Dietz P. and Leigh D., "DiamondTouch: A multi-user touch technology", Proceedings of UIST 2001, Orlando, Florida, USA, pp. 219-226.

[7] Gavaghan, D., Lloyd, S., Boyd, D.R.S., Jeffreys, P.W., Simpson, A., MacRandal, D.F., Sastry, L. and Kleese van Dam, K. Integrative Biology - exploiting e-Science to combat fatal diseases. Proceedings of UK e-Science All Hands Meeting 2004.

[8] Geddes J., Lloyd S., Simpson A., Rossor M., Fox N., Hill D., Hajnal J., Lawrie S., McIntosh A., Johnstone E., Wardlaw J., Perry D., Procter R., Bath P., and Bullmore E. ,NeuroGrid: "Collaborative Neuroscience via Grid Computing". Proceedings All Hands Meeting 2005, Nottingham

[9] Goble C., Wroe C, Stevens R and the myGrid consortium. "Grid Project: Services, Architecture and Demonstrator". Proceedings of UK e-Science All Hands Meeting 2003, Nottingham, UK, September 2003.

[10] KITWARE INC (no date). Insight Segmentation and Registration Toolkit [online]. [cited August $8^{\text {th }}$, 2008].<http://www.itk.org/>.

[11] KITWARE INC (no date). VTK Home Page [online]. [cited August $8^{\text {th }}, 2008$ ].<http://www.vtk.org/>.

[12] MICROSOFT CORPORATION (2007). "Microsoft ${ }^{\circledR}$ Surface" [online]. [cited 6th February 2008]. <http://www.microsoft.com/surface/>.

[13] MICROSOFT CORPORATION (2008). "Windows Workflow Foundation".[online][cited August 13 ${ }^{\text {th }}, 2008$ ]. $<\mathrm{http}: / / \mathrm{msdn}$.microsoft.com/enus/netframework/aa663328.aspx>

[14] MyExperiment (2008). "Main Page My ExperimentWiki" [online]. [cited 30th January 2008]. <http://wiki.myexperiment.org/index.php/Main_Page〉.

[15] UNIVERSITY OF UTAH (no date). "SciRun scientific Computing and Imaging Institute". [online], [cited February $6^{\text {th }} 2008$ ]

<http://software.sci.utah.edu/scirun.html>.

[16] Simpson A., Power D., Slaymaker M., and Politou E. "GIMI: Generic Infrastructure for Medical Informatics" Proceedings of the 18th IEEE Symposium on ComputerBased Medical Systems (CBMS'05),2005

[17] Oinn T., Addis M., Ferris J., Marvin D., Senger M., Greenwood M., Carver T., Glover K., Pocock M.R., Wipat A. and Li P. "Taverna: a tool for the composition and enactment of bioinformatics workflows" Bioinformatics. vol. 20-17, pp. 3045-3054. Oxford University Press. 2004.

[18] Prior F.W., Erickson B.J.,Tarbox L.Open Source Software Projects of the caBIG ${ }^{\mathrm{TM}}$ In Vivo Imaging Workspace Software Special Interest Group. Journal of Digital Imaging. pp.94-100,2007.

[19] Rowland A.L., Burns M., Hartkens T., Hajnal J.V., Rueckert D., Hill D.L.G. "Information eXtraction from Images (IXI): Image Processing Workflows Using A Grid Enabled Image Database" MICCAI 2004, DiDaMIC Workshop.

[20] Smith L.S., Austin J., Baker S., Borisyuk R., Eglen S., Feng J., Gurney K., Jackson T., Kaiser M., Overton P., Panzeri S., Quian Quiroga R., Schultz S.R., Sernagor E., Smith V.A., Smulders T.V., Stuart L., Whittington M., Ingram C.'The CARMEN e-Science pilot project: Neuroinformatics work packages". In Proc of the UK eScience All Hands Meeting 2007, pp. 591-598, 2007.

[21] The Numerical Algorithms Group (2008). "Numerical Algorithms Group IRIS Explorer"[online]. [cited August $5^{\text {th }}$, 2008]. <http://www.nag.co.uk/welcome_iec.asp>.

[22] Yushkevich P.A., Piven J., Hazlett H.c., Smith R.G., Ho S., Gee J.C. and G. Gerig. "User-guided 3D active contour segmentation of anatomical structures: Significantly improved efficiency and reliability" Neuroimage vol. 31, pp. 1116-1128. 2006. 